\title{
Treatment-related damage in elderly-onset ANCA-associated vasculitis: safety outcome analysis of two nationwide prospective cohort studies
}

Ken-Ei Sada ${ }^{1,2^{*}}$, Keiji Ohashi ${ }^{1}$, Yosuke Asano ${ }^{1}$, Keigo Hayashi ${ }^{1}$, Michiko Morishita ${ }^{1}$, Haruki Watanabe ${ }^{1}$, Yoshinori Matsumoto ${ }^{1}$, Shouichi Fujimoto ${ }^{3}$, Yoshinari Takasaki ${ }^{4}$, Kunihiro Yamagata ${ }^{5}$, Shogo Banno ${ }^{6}$, Hiroaki Dobashi ${ }^{7}$, Koichi Amano ${ }^{8}$, Masayoshi Harigai ${ }^{9}$, Yoshihiro Arimura ${ }^{10,11}$, Hirofumi Makino ${ }^{12}$ and the Japan Research Committee of the Ministry of Health, Labour, and Welfare for Intractable Vasculitis (JPVAS) and the Research Committee of Intractable Renal Disease of the Ministry of Health, Labour, and Welfare of Japan

\begin{abstract}
Background: It is not elucidated that there is treatment-related damage in elderly patients with antineutrophil cytoplasmic antibody (ANCA)-associated vasculitis (AAV).

Methods: Elderly ( $\geq 75$ years of age) patients were enrolled from two nationwide prospective inception cohort studies. The primary outcome was 12-month treatment-related Vasculitis Damage Index (VDI) score. Secondary outcomes included serious infections within 6 months, total VDI score, remission, and relapse. Patient characteristics and outcomes were compared across three different initial glucocorticoid (GC) dose groups: high-dose, prednisolone (PSL) $\geq 0.8 \mathrm{mg} / \mathrm{kg} /$ day; medium-dose, $0.6 \leq \mathrm{PSL}<0.8 \mathrm{mg} / \mathrm{kg} /$ day; and low-dose, $\mathrm{PSL}<0.6 \mathrm{mg} / \mathrm{kg} /$ day.

Results: Of the 179 eligible patients, the mean age was 80.0 years; 111 (62\%) were female. The mean Birmingham Vasculitis Activity Score was 16.1. Myeloperoxidase-ANCA findings were positive in 168 (94\%) patients, while proteinase 3-ANCA findings were positive in 11 (6\%). The low-dose group was older and had higher serum creatinine levels than the other groups. There were no statistically significant intergroup differences in remission or relapse, whereas serious infection developed more frequently in the high-dose (29 patients [43\%]) than the lowdose (13 patients [22\%]) or medium-dose (10 patients [19\%]) groups $(p=0.0007)$. Frequent VDI items at 12 months included hypertension (19\%), diabetes (13\%), atrophy and weakness (13\%), osteoporosis (8\%), and cataracts (8\%). Logistic regression analysis revealed that GC dose at 12 months (odds ratio, 1.14; 95\% confidence interval, 1.00-1.35) was a predictor for diabetes.

(Continued on next page)
\end{abstract}

\footnotetext{
*Correspondence: sadak@kochi-u.ac.jp

'Department of Clinical Epidemiology, Kochi Medical School, Kochi

University, Kohasu, Oko-cho, Nankoku 783-8505, Japan

2Department of Nephrology, Rheumatology, Endocrinology, and Metabolism,

Okayama University Graduate School of Medicine, Dentistry, and

Pharmaceutical Sciences, Okayama, Japan

Full list of author information is available at the end of the article
}

(c) The Author(s). 2020 Open Access This article is licensed under a Creative Commons Attribution 4.0 International License, which permits use, sharing, adaptation, distribution and reproduction in any medium or format, as long as you give appropriate credit to the original author(s) and the source, provide a link to the Creative Commons licence, and indicate if changes were made. The images or other third party material in this article are included in the article's Creative Commons licence, unless indicated otherwise in a credit line to the material. If material is not included in the article's Creative Commons licence and your intended use is not permitted by statutory regulation or exceeds the permitted use, you will need to obtain permission directly from the copyright holder. To view a copy of this licence, visit http://creativecommons.org/licenses/by/4.0/. The Creative Commons Public Domain Dedication waiver (http://creativecommons.org/publicdomain/zero/1.0/) applies to the data made available in this article, unless otherwise stated in a credit line to the data. 
(Continued from previous page)

Conclusion: A reduced initial GC dose with rapid reduction might be required to ensure the safe treatment of elderly AAV patients.

Keywords: ANCA-associated vasculitis, Chronic damage, Elderly patients, Glucocorticoids

\section{Introduction}

Treatment with high-dose glucocorticoid (GC) and immunosuppressants has greatly improved the prognosis of patients with antineutrophil cytoplasmic antibody (ANCA)-associated vasculitis (AAV) $[1,2]$, but chronic damage has become a major concern in such patients. Because disease severity was reportedly related to chronic damage [3], intensive immunosuppressive treatment is required to induce the remission of AAV. On the other hand, $\mathrm{GC}$ is a risk factor for chronic damage as well as infectious complications $[4,5]$.

AAV often occurs in elderly populations, particularly in Japan [6], and aging is a strong risk factor for death and end-stage renal disease [7-10]. Due to the high incidence of chronic conditions including diabetes mellitus, osteoporosis, cataracts, and hypertension in elderly populations with AAV [11], GC-related damage might be more serious. Because many studies of AAV have excluded elderly patients, optimization of the initial GC dose and tapering speed might be required to ensure their safe treatment.

In this study, treatment-related damage was evaluated in patients with elderly-onset AAV based on safety outcome analysis using data from two nationwide prospective inception cohort studies.

\section{Methods}

\section{Database}

We used datasets from two cohort studies: RemIT-JAV (observational cohort study of remission induction therapy in Japanese patients with ANCA-associated vasculitis [UMIN000001648]) and RemIT-JAV-RPGN (observational cohort study of remission induction therapy in Japanese patients with ANCA-associated vasculitis and rapidly progressive glomerulonephritis [UMIN000005136]). Consecutive patients with newly diagnosed AAV were enrolled in RemIT-JAV between April 2009 and December 2010 from 22 tertiary care institutions or in RemIT-JAV-RPGN from April 2011 to March 2014 from 53 tertiary care institutions. The criteria for enrolment for both studies included the following: (1) diagnosis of AAV by the site investigators, (2) fulfillment of criteria for primary systemic vasculitis as proposed by the European Medicines Agency algorithm [12], and (3) starting immunosuppressive treatment based on site investigator discretion $[13,14]$. The exclusion criteria were as follows: (1) age younger than 20 years, (2) serologic evidence of hepatitis B or C virus infection, and (3) a history of malignancy. Baseline data recorded for each patient included demographic information, laboratory data, Birmingham Vasculitis Activity Score (BVAS) 2003 [15], and disease severity. Disease severity was subclassified as localized, early systemic, generalized, or severe according to the European Vasculitis Study Group definition of disease severity types [16]. Patients with threatened vital organ function were classified as having generalized disease, while those with organ failure were classified as having severe disease. Detailed definitions of disease severity were described previously [17]. Patients were evaluated at $3,6,12,18$, and 24 months and at relapse, and the following data were collected: vital status, BVAS 2003, laboratory data, treatments, and adverse events. The Vasculitis Damage Index (VDI) score was recorded at 6, 12, and 24 months [18].

\section{Patient selection and outcome measures}

In the present study, patients with elderly-onset $(\geq 75$ years) AAV were enrolled. Patients for whom data about the GC dose were lacking were excluded.

The primary outcome measure was 12-month treatment-related VDI score. Treatment-related VDI was defined as in the previous report as follows: osteoporosis, diabetes, cataracts, atrophy and weakness, malignancy, gonadal failure, marrow failure, chemical cystitis, avascular necrosis, hypertension, angina/coronary artery disease, alopecia, cerebrovascular accident, myocardial infarction, and mouth ulcers [4]. Secondary outcome measures included serious infections within 6 months, 12-month total VDI score, remission, and relapse. Our definition of serious infections was based on an International Conference on Harmonization report [19]. Bacterial infections requiring intravenous antibiotic administration and opportunistic infections were considered serious infections. The diagnosis of infection was based on the attending physician's clinical diagnosis using a comprehensive evaluation of physical findings, laboratory data, and imaging data. Remission was defined as a BVAS score of 0 on 2 consecutive occasions at least 1 month apart according to the European League Against Rheumatism (EULAR) recommendations [16]. Relapse was defined as recurrent or new-onset clinical signs and symptoms attributable to active vasculitis as we previously reported [20]. 


\section{Statistical analysis}

Clinical characteristics are presented as mean \pm standard deviation (SD). The patients were divided into three groups according to initial GC dose: high-dose, prednisolone (PSL) $\geq 0.8 \mathrm{mg} / \mathrm{kg} /$ day; medium-dose, $0.6 \leq \mathrm{PSL}<$ $0.8 \mathrm{mg} / \mathrm{kg} /$ day; and low-dose, PSL $<0.6 \mathrm{mg} / \mathrm{kg} /$ day. Patient characteristics and outcomes were compared across the three GC dose groups. Continuous variables were compared using the Mann-Whitney $U$ test, whereas categorical variables were compared between two groups using the Fisher exact probability test. $p$ values $<0.05$ were considered significant. Statistical significance was determined using Bonferroni correction, $<0.05 / 3$, to adjust for multiple testing. To explore the factors related to VDI items, multiple linear regression and logistic regression analyses were performed. All statistical analyses were performed using JMP 11.2.0 software (SAS Institute Inc., Cary, NC, USA).

\section{Results}

\section{Patient characteristics}

Of 477 patients registered in the two cohort studies, 181 fulfilled the inclusion criteria. Among them, 2 were excluded because of a lack of data about the GC dose. For the analysis of VDI score, 60 patients without a 12month score were excluded. Of those 60 excluded patients, 19 died by 12 months. The mean age (SD) of the 179 enrolled patients was 80.0 (3.8) years, $111(62 \%)$ were female, and the baseline mean (SD) BVAS was 16.1 (6.6). Seven (4\%) patients had eosinophilic granulomatosis with polyangiitis, 28 (16\%) had granulomatosis with polyangiitis, $113(63 \%)$ had microscopic polyangiitis, and 31 (17\%) were unclassifiable. Myeloperoxidase-antineutrophil cytoplasmic antibody (MPO-ANCA) results were positive in 168 (94\%) patients, while proteinase 3-ANCA (PR3-ANCA) results were positive in $11(6 \%)$ patients. Concomitant cyclophosphamide $(\mathrm{CY})$ was used in 54 (30\%) patients, and the mean (SD) GC dose was 0.73 (0.25) $\mathrm{mg} / \mathrm{kg} /$ day. Prophylaxis against Pneumocystis pneumonia was administrated in 153 of 178 (86\%) patients (there was no statistical difference compared to patients aged < 75 years: 243 of 292 [83\%], $p=0.43$ ).

The patient characteristics of the three groups are summarized in Table 1. The low-dose group had a significantly higher mean age than the high-dose group. There were no significant intergroup differences in sex, disease classification, or disease severity. Mean BVAS score did not differ significantly, while the high-dose

Table 1 Patient characteristics by initial GC dose group

\begin{tabular}{|c|c|c|c|}
\hline & Low-dose $(n=59)$ & Medium-dose $(n=52)$ & High-dose $(n=68)$ \\
\hline Male/female & $27 / 32$ & $18 / 34$ & $23 / 45$ \\
\hline Age (years), mean $\pm S D\left(\right.$ median) ${ }^{\dagger}$ & $80.9 \pm 3.9(80)$ & $79.9 \pm 3.6(80)$ & $79.1 \pm 3.6(78)$ \\
\hline \multicolumn{4}{|l|}{ Disease classification, $n$} \\
\hline EGPA & 2 & 2 & 3 \\
\hline GPA & 5 & 9 & 14 \\
\hline MPA & 42 & 33 & 38 \\
\hline Unclassifiable & 10 & 8 & 13 \\
\hline \multicolumn{4}{|l|}{ Disease severity, n (\%) } \\
\hline Localized & 1 & 3 & 3 \\
\hline Early systemic & 12 & 14 & 18 \\
\hline Systemic & 34 & 27 & 35 \\
\hline Severe & 12 & 8 & 12 \\
\hline BVAS, mean $\pm S D$ & $15.8 \pm 6.3$ & $17.3 \pm 6.0$ & $15.4 \pm 7.2$ \\
\hline MPO-ANCA-positive, $n$ (\%) & $55(93)$ & $49(94)$ & $64(94)$ \\
\hline PR3-ANCA-positive, $n$ (\%) & 0 & $2(4)$ & $3(4)$ \\
\hline Serum creatinine $(\mathrm{mg} / \mathrm{dL})$, mean $\pm \mathrm{SD}^{\dagger}$ & $3.11 \pm 3.56$ & $2.36 \pm 2.15$ & $1.59 \pm 1.31$ \\
\hline Interstitial lung disease, $n$ (\%) & $29(49)$ & $24(46)$ & $29(43)$ \\
\hline \multicolumn{4}{|l|}{ Treatment } \\
\hline Initial daily dose of PSL (mg/kg/day), mean $\pm \mathrm{SD}^{\#,+}$ & $0.47 \pm 0.10$ & $0.69 \pm 0.05$ & $0.98 \pm 0.18$ \\
\hline Cyclophosphamide, $n$ (\%) & $8(13)$ & $10(19)$ & $36(53)$ \\
\hline
\end{tabular}

Comparisons among groups were made using the Mann-Whitney $U$ test or Fisher's exact probability test. Statistical significance was determined by using Bonferroni correction $(<0.05 / 3)$. ANCA antineutrophil cytoplasmic antibody, BVAS Birmingham Vasculitis Activity Score, EGPA eosinophilic granulomatosis with polyangiitis, GPA granulomatosis with polyangiitis, MPA microscopic polyangiitis, MPO myeloperoxidase, PR3 proteinase 3, PSL prednisolone, SD standard deviation "Medium-dose vs. high-dose group

${ }^{\dagger}$ Low-dose vs. high-dose group 
group exhibited a significantly lower mean serum creatinine level than the low-dose group.

Mean (SD) daily PSL doses at initial treatment in the low-, medium-, and high-dose groups were $0.47(0.10)$, $0.69(0.05)$, and $0.98(0.18) \mathrm{mg} / \mathrm{kg}$, respectively. The proportion of concomitant CY use was significantly different among groups: low-dose, 8 (13\%); medium-dose, 10 (19\%); and high-dose, 36 (53\%); $p<0.0001$.

\section{Treatment effectiveness and safety}

By 12 months, 151 (84\%) patients achieved remission; of those, $17(11 \%)$ experienced a relapse. Mean (SD) total VDI score at 12 months was 2.4 (1.8), while mean (SD) treatment-related VDI score was 0.7 (1.0). The proportions of patients with each VDI item at 12 months are shown in Fig. 1. Frequent VDI items ( $>5 \%$ of enrolled patients) were as follows: hypertension (19\%), diabetes (13\%), atrophy and weakness (13\%), osteoporosis (8\%), and cataracts (8\%). Serious infections within 6 months developed in $52(29 \%)$ patients.

There were no statistically significant intergroup differences in remission or relapse by 12 months: remission-50 (85\%) of 59 patients in low-dose, 41 (79\%) of 52 in medium-dose, and 60 (88\%) of 68 in high-dose, respectively, $p=0.38$; relapse $-5(10 \%)$ of 50 in low-dose, 5 (12\%) of 41 in medium-dose, and 7 (12\%) of 60 in highdose, respectively, $p=0.93$. Even limited in patients with severe form, remission and relapse were not different statistically (remission: 66\% in low-dose, 63\% in medium-dose, and $67 \%$ in high-dose, respectively, $p=$ 0.98 ; relapse: $0 \%, 20 \%$, and $13 \%$, respectively, $p=0.46$ ). Serious infections developed more frequently in the high-dose $(n=29[43 \%])$ than in the low-dose $(n=13$ [22\%]) or medium-dose $(n=10$ [19\%]) groups ( $p=$ 0.0007). Same trends were found in each dataset (RemIT-JAV group: $45 \%$ in high-dose, $13 \%$ in mediumdose, and $15 \%$ in low-dose, respectively, $p=0.04$; RemIT-JAV-RPGN group: $41 \%$ in high-dose, $22 \%$ in medium-dose, and $24 \%$ in low-dose, respectively, $p=$ $0.10)$.

\section{Vasculitis Damage Index}

Mean (SD) VDI scores of all patients in the RemIT-JAV and RemIT-JAV-RPGN groups were 1.7 (1.7) and 2.1 (1.6) at 6 months, and 2.0 (1.7) and 2.5 (1.8) at 12 months, respectively.

Of 121 patients with VDI scores at 12 months, total and treatment-related VDI scores at 12 months showed no statistically significant intergroup differences (total VDI score: low-dose, $2.65 \pm 1.99$; medium-dose, $2.40 \pm$ 1.67; and high-dose, $2.25 \pm 1.70, p=0.57$; treatmentrelated VDI score: low-dose, $0.91 \pm 1.18$; medium-dose, $0.45 \pm 0.71$; and high-dose, $0.77 \pm 1.04, p=0.14$ ).

For the determination of variables associated with total and treatment-related VDI scores at 12 months, a multiple linear regression analysis was performed of the following candidate variables: sex, age, initial GC dose, initial CY usage, and initial serum creatinine level. No

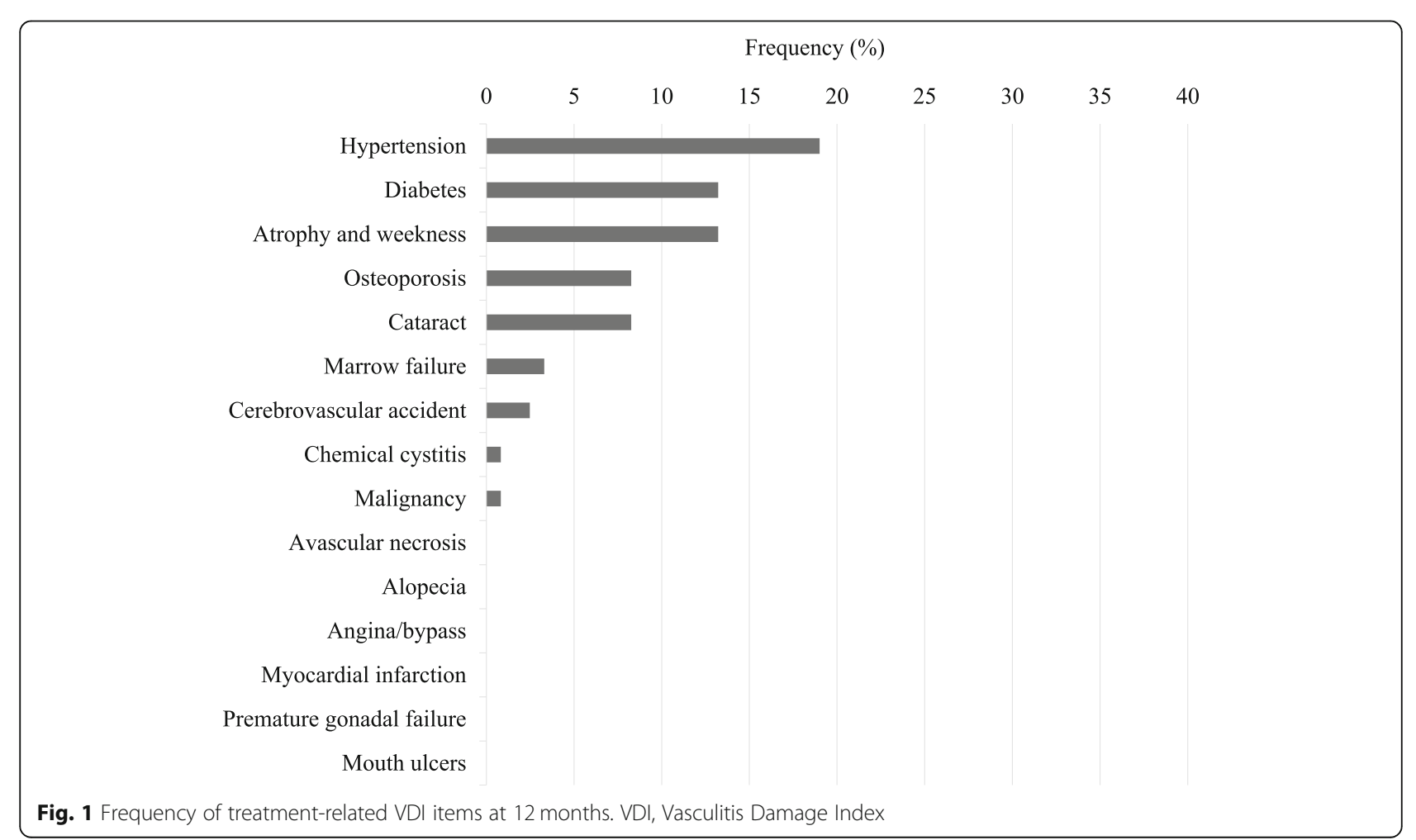


statistically significant variables were identified (Supplementary Table 1).

Next, we explored variables related to 5 frequent treatment-related VDI items at 12 months using logistic regression analysis including the same candidate variables. Hypertension, atrophy and weakness, osteoporosis, and cataracts were eliminated (Supplementary Table 2), while $\mathrm{CY}$ usage was identified as an independent predictor for a less frequent development of diabetes (Table 2, model 1).

Because mean (SD) GC dose at 12 months was lower in patients with concomitant $\mathrm{CY}$ than in those without it (6.8 [3.5] vs. 9.5 [6.9] $\mathrm{mg} /$ day, $p=0.016$ ), we performed a multiple linear regression analysis including GC dose at 12 months instead of concomitant CY usage; GC dose at 12 months was also identified as an independent predictor for diabetes (Table 2, model 2).

\section{Discussion}

This is the first report to evaluate damage in elderly patients with AAV. The low-dose initial GC group was older and had more severe renal impairment but less frequent concomitant $\mathrm{CY}$ use. The high-dose group more frequently developed serious infections. Total and treatment-related VDI scores did not differ significantly among the initial GC dose groups. GC dose at 12 months was an independent predictor for diabetes.

Our findings suggest that initial GC dose perhaps is reduced during treatment in elderly patients with AAV. Serious infections developed more frequently in the high-dose group, but remission, relapse, and VDI score were comparable to those of the other groups. Although $\mathrm{CY}$ dose was recommended to be reduced according to age and renal function with concern about adverse drug reactions in the EULAR recommendation for the

Table 2 Risk factors for the development of diabetes at 12 months

\begin{tabular}{lll}
\hline & Odds ratio (95\% Cl) & $\boldsymbol{p}$ value \\
\hline Model 1 & $1.06(0.93-1.24)$ & 0.36 \\
Age, years & $0.73(0.24-2.01)$ & 0.55 \\
Female sex & $0.83(0.66-0.99)$ & 0.040 \\
Serum creatinine, mg/dL & $3.32(0.29-41.66)$ & 0.34 \\
Initial PSL dose, mg/kg/day & $0.29(0.09-0.87)$ & 0.027 \\
Concomitant cyclophosphamide use & \\
Model 2 & $1.08(0.94-1.26)$ & 0.28 \\
Age, years & $0.76(0.25-2.09)$ & 0.60 \\
Female sex & $0.86(0.70-1.02)$ & 0.09 \\
Serum creatinine, mg/dL & $1.26(0.13-14.65)$ & 0.85 \\
Initial PSL dose, mg/kg/day & $1.14(1.00-1.35)$ & 0.045 \\
PSL dose at 12 months, mg/day & & \\
\hline Cl confidential interval, PSL prednisolone &
\end{tabular}

treatment of AAV [21], the initial dose adjustment of GC was not stated, and PSL $1 \mathrm{mg} / \mathrm{kg} /$ day has traditionally been initiated since the 1970s [22]. The PEXIVAS trial reported that a reduced-dose GC regimen (initial dose, $\sim 0.5 \mathrm{mg} / \mathrm{kg} /$ day) was non-inferior to the standard regimen (initial dose, $\sim 1 \mathrm{mg} / \mathrm{kg} /$ day) with respect to death or end-stage renal failure in patients with severe AAV [23]. Initial GC dose and age were reportedly related to serious infection in the immunosuppressive treatment of rheumatic diseases [24-26]. Our previous study also showed that PSL $>0.8 \mathrm{mg} / \mathrm{kg} /$ day was a risk factor for severe infection in patients with AAV [5]. Therefore, the reduction of the initial GC dose could lead to improved safety outcomes in elderly patients.

Diabetes, atrophy and weakness, osteoporosis, and cataracts were emphasized as chronic damage in elderly AAV patients. Although the order of the frequency of treatment-related VDI items was comparable to those of the previous report for VDI for AAV patients of all ages, diabetes $(13 \%$ in present study vs. $8 \%$ in the previous study), atrophy and weakness ( $13 \%$ vs. $6 \%)$, osteoporosis ( $8 \%$ vs. $4 \%)$, and cataracts ( $8 \%$ vs. $3 \%)$ were more frequent in the present study [27]. These items are also well-known concerns in general elderly patients, and our result indicates that $\mathrm{AAV}$ treatment accelerates this damage in elderly AAV patients.

A delay in the GC reduction without concomitant $\mathrm{CY}$ usage and renal impairment might lead to the development of diabetes. Concomitant CY usage was determined as a protective predictor for diabetes by analysis of initial treatment with patient characteristics. Our recent study also demonstrated a GC sparing effect of concomitant $\mathrm{CY}$ in AAV patients, as the GC dose at 12 months was lower in patients with concomitant $\mathrm{CY}$ than those without CY [28]. Even on multivariate analysis with the model including GC dose at 12 months instead of concomitant CY usage, GC dose at 12 months remained a statistical predictor for diabetes. Because the achievement of PSL $5-7.5 \mathrm{mg} /$ day by 5 months is recommended in the EULAR guidelines, an earlier reduction of GC dose might be attempted in elderly AAV patients with renal impairment [21].

There are some limitations to this study. First, the majority of our patients were MPO-ANCA-positive. There are several differences in characteristics between patients with MPO-ANCA and those with PR3-ANCA [29, 30], and our results might not be applicable to the treatment of elderly patients with PR3-ANCA. Second, the treatment strategy was decided by each attending physician, so frail patients might have been treated conservatively, leading to underestimation of the outcomes. Nevertheless, patients with higher GC doses at 12 months had diabetes more frequently, supporting the relevance of a sufficient and early GC dose reduction in elderly 
patients. Third, we could not validate our exploratory results in another dataset because of insufficient sample size, so further validation study is required.

\section{Conclusion}

Rapidly reducing the initial GC dose to $\mathrm{PSL}<0.8 \mathrm{mg} / \mathrm{kg} /$ day perhaps is required to ensure the safe treatment of elderly patients with AAV.

\section{Supplementary information}

Supplementary information accompanies this paper at https://doi.org/10. 1186/s13075-020-02341-6.

Additional file 1: Table S1. Risk factors for total and treatment-related VDI. Table S2. Risk factors for hypertension, atrophy and weakness, cataract, and osteoporosis.

\section{Abbreviations}

AAV: Antineutrophil cytoplasmic antibody-associated vasculitis; ANCA: Antineutrophil cytoplasmic antibody; BVAS: Birmingham Vasculitis Activity Score; CY: Cyclophosphamide; EULAR: European League Against Rheumatism; GC: Glucocorticoid; MPO-ANCA: Myeloperoxidaseantineutrophil cytoplasmic antibody; PR3-ANCA: Proteinase 3-antineutrophil cytoplasmic antibody; PSL: Prednisolone; RemIT-JAV: Observational cohort study of remission induction therapy in Japanese patients with antineutrophil cytoplasmic antibody-associated vasculitis; RemIT-JAVRPGN: Observational cohort study of remission induction therapy in Japanese patients with antineutrophil cytoplasmic antibody-associated vasculitis and rapidly progressive glomerulonephritis; SD: Standard deviation; VDI: Vasculitis Damage Index

\section{Acknowledgements}

The authors thank all the medical staff members in our department. Japan Research Committee of the Ministry of Health, Labour, and Welfare for Intractable Vasculitis (JPVAS) and Research Committee of Intractable Renal Disease of the Ministry of Health, Labour, and Welfare of Japan-in addition to the authors, the following investigators and institutions participated in this study: Department of Nephrology, Faculty of Medicine, University of Tsukuba (Joichi Usui); Department of Rheumatology, Endocrinology and Nephrology, Faculty of Medicine and Graduate School of Medicine, Hokkaido University (Tatsuya Atsumi); Department of Medicine and Rheumatology, Tokyo Metropolitan Geriatric Hospital (Takahiko Sugihara); Department of Nephrology, Internal Medicine, Nagoya University Graduate School of Medicine (Seiichi Matsuo): Department of Human Resource Development of Dialysis Therapy for Kidney Disease, Okayama University Graduate School of Medicine, Dentistry and Pharmaceutical Sciences (Hitoshi Sugiyama); Faculty of Health Sciences, Hokkaido University (Akihiro Ishizu); Department of the Control for Rheumatic Diseases, Graduate School of Medicine, Kyoto University (Takao Fujii); Department of Pathology, Keio University School of Medicine (Yasunori Okada); Department of Respiratory Medicine, Toho University Omori Medical Center (Sakae Homma); Department of Nephrology, Internal Medicine, Nagoya University Graduate School of Medicine (Naotake Tsuboi); Department of Clinical Pathology and Immunology, Kobe University Graduate School of Medicine (Shunichi Kumagai); Department of Nephrology and Dialysis, Kitano Hospital, Tazuke Kofukai Medical Research Institute (Eri Muso); Department of Rheumatology, Shimane University Faculty of Medicine (Yohko Murakawa); Division of Rheumatology, Department of Medical Oncology and Immunology, Nagoya City University Graduate School of Medical Science (Shogo Banno); Department of Hematology, Clinical Immunology and Infectious Diseases, Ehime University Graduate School of Medicine (Hitoshi Hasegawa); Division of Nephrology, Department of Internal Medicine, Jichi Medical University (Wako Yumura); Department of Cardiovascular Medicine, Kyoto Prefectural University School of Medicine (Hiroaki Matsubara); Division of Nephrology, Tokyo Medical University Hachioji Medical Center (Masaharu Yoshida); Department of Dermatology, Kitasato University School of Medicine (Kensei Katsuoka); Division of Immunology and Rheumatology, Department of
Internal Medicine 3, Hamamatsu University School of Medicine (Noriyoshi Ogawa); Department of Hematology, Oncology, Nephrology, and Rheumatology, Akita University Graduate School of Medicine (Atsushi Komatsuda); Department of Rheumatology, Niigata Rheumatic Center (Satoshi Ito); Department of Immunology and Rheumatology, Division of Advanced Preventive Medical Sciences, Nagasaki University Graduate School of Biomedical Sciences (Atsushi Kawakami); Department of Nephrology, Iwate Prefectural Central Hospital (Izaya Nakaya); Division of Nephrology and Rheumatology, Department of Internal Medicine, Fukuoka University School of Medicine (Takao Saito); Shimane University, Faculty of Medicine, Division of Nephrology (Takafumi Ito); Department of Hemodialysis and Apheresis, Yokohama City University Medical Center (Nobuhito Hirawa); Center for Rheumatology, Okayama Saiseikai General Hospital (Masahiro Yamamura); Department of Medical Technology, School of Health Sciences, Faculty of Medicine, Niigata University (Masaaki Nakano); Department of Medicine, Kidney Center, Tokyo Women's Medical University (Kosaku Nitta); Division of Nephrology and Hypertension, Kashiwa Hospital, Jikei University (Makoto Ogura); Department of Respiratory Medicine, Allergy and Clinical Immunology, Nagoya City University Graduate School of Medical Sciences (Taio Naniwa); Division of Rheumatology and Allergology, Department of Internal Medicine, St. Marianna University School of Medicine (Shoichi Ozaki); Department of Nephrology and Endocrinology, Graduate School of Medicine, The University of Tokyo (Junichi Hirahashi); Division of Kidney and Hypertension, Department of Internal Medicine, Jikei University School of Medicine (Tatsuo Hosoya); Department of Nephrology and Laboratory Medicine, Faculty of Medicine, Institute of Medical, Pharmaceutical and Health Sciences, Kanazawa University (Takashi Wada); Division of Nephrology, Department of Internal Medicine, Juntendo University Faculty of Medicine (Satoshi Horikoshi); Institute of Rheumatology, Tokyo Women's Medical University (Yasushi Kawaguchi); Division of Clinical Immunology, Graduate School of Comprehensive Human Sciences, University of Tsukuba (Taichi Hayashi); Department of Nephrology, Hypertension, Diabetology, Endocrinology and Metabolic, Fukushima Medical University (Tsuyoshi Watanabe); Department of Nephrology, Japanese Red Cross Nagoya Daini Hospital (Daijo Inaguma); Department of Integrated Therapy for Chronic Kidney Disease, Kyushu University (Kazuhiko Tsuruya); Niigata Prefectural Shibata Hospital (Noriyuki Homma); Division of Rheumatology, Department of Internal Medicine, Keio University School of Medicine (Tsutomu Takeuchi); Division of Cardiology, Nephrology, Pulmonology and Neurology, Department of Internal Medicine, Asahikawa Medical University (Naoki Nakagawa); Kurobe City Hospital (Shinichi Takeda); National Fukuoka Higashi Medical Center (Ritsuko Katafuchi); Division of Nephrology, Department of Medicine, Faculty of Medical Sciences, University of Fukui (Masayuki Iwano); and Tokyo Medical University Ibaraki Medical Center (Masaki Kobayashi).

\section{Authors' contributions}

$\mathrm{KS}$ and $\mathrm{KO}$ were responsible for the conception and design, analysis, and manuscript writing. $\mathrm{YA}, \mathrm{KH}, \mathrm{MM}, \mathrm{HW}$, and $\mathrm{YM}$ were responsible for the data collection and critical revision. SF, YT, KY, SB, HD, and KA were responsible for the data collection and interpretation. $M H, Y A$, and $H M$ were responsible for the data collection, interpretation, and critical revision. YA was responsible for the conception and design, data collection and analysis, and critical revision. HM was responsible for the data analysis, interpretation, and critical revision. The authors read and approved the final manuscript.

\section{Funding}

This work was supported by grants from the Ministry of Health, Labour, and Welfare, Japan (nannti-ippann-004 and nannti-ippann-018), and the Japan Agency for Medical Research and Development (JP17ek0109104,

JP17ek0109121, and JP18ek0109360).

\section{Availability of data and materials}

The dataset analyzed in this paper is available from the corresponding author on reasonable request.

\section{Ethics approval and consent to participate}

This study was conducted in accordance with the Declaration of Helsinki and the ethical guidelines for epidemiological research in Japan. This study was approved by the Ethics Committee of Okayama University Hospital and Graduate School of Medicine, Dentistry, and Pharmaceutical Sciences 
(approval number, Ken1904-023). Informed consent and permission to publish their data were obtained from all participants.

\section{Consent for publication}

No individual person's data were presented in any form in this study, and therefore, no consent to publish is required.

\section{Competing interests}

KS received speaker's fee from Astra Zeneca K.K. KA received speaker's fee from Chugai Pharmaceutical Co. $\mathrm{MH}$ has received unrestricted research grants from AbbVie Japan GK; Ayumi Pharmaceutical Co.; Bristol Myers Squibb Co., Ltd.; Eisai Co., Ltd.; Nippon Kayaku Co., Ltd.; Mitsubishi Tanabe Pharma Co.; and Teijin Pharma Ltd. MH has received speaker's fee from Boehringer-Ingelheim; Kissei Pharmaceutical Co., Ltd.; Eli Lilly Japan K.K.; and Chugai Pharmaceutical Co., Ltd. MH is a consultant for AbbVie; BoehringerIngelheim; Kissei Pharmaceutical Co., Ltd.; and Teijin Pharma. HM is a consultant for AbbVie, Boehringer-Ingelheim, and Teijin.

\section{Author details}

'Department of Clinical Epidemiology, Kochi Medical School, Kochi University, Kohasu, Oko-cho, Nankoku 783-8505, Japan. ${ }^{2}$ Department of Nephrology, Rheumatology, Endocrinology, and Metabolism, Okayama University Graduate School of Medicine, Dentistry, and Pharmaceutical Sciences, Okayama, Japan. ${ }^{3}$ Department of Hemovascular Medicine and Artificial Organs, Faculty of Medicine, University of Miyazaki, Miyazaki, Japan. ${ }^{4}$ Department of Internal Medicine and Rheumatology, Juntendo University School of Medicine, Tokyo, Japan. ${ }^{5}$ Department of Nephrology, Faculty of Medicine, University of Tsukuba, Ibaraki, Japan. ${ }^{6}$ Department of Nephrology and Rheumatology, Aichi Medical University, Nagakute, Japan. ${ }^{7}$ Division of Hematology, Rheumatology and Respiratory Medicine, Department of Internal Medicine, Faculty of Medicine, Kagawa University, Kagawa, Japan. ${ }^{8}$ Department of Rheumatology and Clinical Immunology, Saitama Medica Center, Saitama Medical University, Kawagoe, Japan. ${ }^{9}$ Department of Rheumatology, Tokyo Women's Medical University School of Medicine, Tokyo, Japan. ${ }^{10}$ Department of Nephrology and Rheumatology, Kyorin University School of Medicine, Tokyo, Japan. ${ }^{11}$ Kichijoji Asahi Hospital, Tokyo, Japan. ${ }^{12}$ Okayama University, Okayama, Japan.

Received: 9 July 2020 Accepted: 2 October 2020

Published online: 12 October 2020

\section{References}

1. Holle JU, Gross WL, Latza U, Nolle B, Ambrosch P, Heller M, Fertmann R, Reinhold-Keller E. Improved outcome in 445 patients with Wegener's granulomatosis in a German vasculitis center over four decades. Arthritis Rheum. 2011;63(1):257-66.

2. Hilhorst M, Wilde B, van Paassen P, Winkens B, van Breda VP, Cohen Tervaert JW. Improved outcome in anti-neutrophil cytoplasmic antibody (ANCA)-associated glomerulonephritis: a 30-year follow-up study. Nephrol Dial Transplant. 2013:28(2):373-9.

3. Sreih AG, Mandhadi R, Aldaghlawi F, Khan A, Irshad V, Finn K, Block JA ANCA-associated vasculitis in Hispanic Americans: an unrecognized severity. Clin Rheumatol. 2015;34(5):943-8.

4. Robson J, Doll H, Suppiah R, Flossmann O, Harper L, Hoglund P, Jayne D, Mahr A, Westman K, Luqmani R. Glucocorticoid treatment and damage in the anti-neutrophil cytoplasm antibody-associated vasculitides: long-term data from the European Vasculitis Study Group trials. Rheumatology (Oxford). 2015;54(3):471-81.

5. Watanabe-Imai K, Harigai M, Sada KE, Yamamura M, Fujii T, Dobashi H, Amano K, Ito S, Homma S, Kumagai S, et al. Clinical characteristics of and risk factors for serious infection in Japanese patients within six months of remission induction therapy for antineutrophil cytoplasmic antibodyassociated vasculitis registered in a nationwide, prospective, inception cohort study. Mod Rheumatol. 2017:27(4):646-51.

6. Fujimoto S, Watts RA, Kobayashi S, Suzuki K, Jayne DR, Scott DG, Hashimoto $\mathrm{H}$, Nunoi $\mathrm{H}$. Comparison of the epidemiology of anti-neutrophil cytoplasmic antibody-associated vasculitis between Japan and the U.K. Rheumatology (Oxford). 2011:50(10):1916-20.

7. Harper L, Savage CO. ANCA-associated renal vasculitis at the end of the twentieth century--a disease of older patients. Rheumatology (Oxford). 2005, 44(4):495-501.
8. Chen M, Yu F, Zhang Y, Zhao MH. Antineutrophil cytoplasmic autoantibodyassociated vasculitis in older patients. Medicine (Baltimore). 2008;87(4):203-9.

9. Bomback AS, Appel GB, Radhakrishnan J, Shirazian S, Herlitz LC, Stokes B, D'Agati VD, Markowitz GS. ANCA-associated glomerulonephritis in the very elderly. Kidney Int. 2011;79(7):757-64.

10. Little MA, Nightingale P, Verburgh CA, Hauser T, De Groot K, Savage C, Jayne D, Harper L, European Vasculitis Study G. Early mortality in systemic vasculitis: relative contribution of adverse events and active vasculitis. Ann Rheum Dis. 2010;69(6):1036-43.

11. Weiner M, Goh SM, Mohammad AJ, Hruskova Z, Tanna A, Bruchfeld A, Selga D, Chocova Z, Westman K, Eriksson P, et al. Outcome and treatment of elderly patients with ANCA-associated vasculitis. Clin J Am Soc Nephrol. 2015:10(7):1128-35.

12. Watts R, Lane S, Hanslik T, Hauser T, Hellmich B, Koldingsnes W, Mahr A, Segelmark M, Cohen-Tervaert JW, Scott D. Development and validation of a consensus methodology for the classification of the ANCA-associated vasculitides and polyarteritis nodosa for epidemiological studies. Ann Rheum Dis. 2007:66(2):222-7.

13. Sada KE, Yamamura M, Harigai M, Fujii T, Dobashi H, Takasaki Y, Ito S, Yamada H, Wada T, Hirahashi J, et al. Classification and characteristics of Japanese patients with antineutrophil cytoplasmic antibody-associated vasculitis in a nationwide, prospective, inception cohort study. Arthritis Res Ther. 2014;16(2):R101.

14. Sada KE, Yamamura M, Harigai M, Fujii T, Arimura Y, Makino H. Issues associated with the Ministry of Health, Labour and Welfare diagnostic criteria for antineutrophil cytoplasmic antibody-associated vasculitides: reclassification of patients in the prospective cohort study of Remission Induction Therapy in Japanese patients with ANCA-associated vasculitides according to the MHLW criteria. Mod Rheumatol. 2015;25(4):657-9.

15. Flossmann $O$, Bacon $P$, de Groot K, Jayne D, Rasmussen N, Seo P, Westman K, Luqmani R. Development of comprehensive disease assessment in systemic vasculitis. Ann Rheum Dis. 2007;66(3):283-92.

16. Hellmich B, Flossmann O, Gross WL, Bacon P, Cohen-Tervaert JW, Guillevin $L$, Jayne D, Mahr A, Merkel PA, Raspe $H$, et al. EULAR recommendations for conducting clinical studies and/or clinical trials in systemic vasculitis: focus on anti-neutrophil cytoplasm antibody-associated vasculitis. Ann Rheum Dis. 2007;66(5):605-17.

17. Sada KE, Harigai M, Amano K, Atsumi T, Fujimoto S, Yuzawa Y, Takasaki Y, Banno S, Sugihara T, Kobayashi M, et al. Comparison of severity classification in Japanese patients with antineutrophil cytoplasmic antibody-associated vasculitis in a nationwide, prospective, inception cohort study. Mod Rheumatol. 2016;26(5):730-7

18. Exley AR, Bacon PA, Luqmani RA, Kitas GD, Gordon C, Savage CO, Adu D. Development and initial validation of the Vasculitis Damage Index for the standardized clinical assessment of damage in the systemic vasculitides. Arthritis Rheum. 1997:40(2):371-80.

19. Vijayananthan A, Nawawi O. The importance of Good Clinical Practice guidelines and its role in clinical trials. Biomed Imaging Interv J. 2008;4(1):e5.

20. Hara A, Wada T, Sada KE, Amano K, Dobashi H, Harigai M, Takasaki Y, Yamada $H$, Hasegawa $H$, Hayashi $T$, et al. Risk factors for relapse of antineutrophil cytoplasmic antibody-associated vasculitis in Japan: a nationwide, prospective cohort study. J Rheumatol. 2018;45(4):521-8.

21. Yates M, Watts RA, Bajema IM, Cid MC, Crestani B, Hauser T, Hellmich B, Holle JU, Laudien M, Little MA, et al. EULAR/ERA-EDTA recommendations for the management of ANCA-associated vasculitis. Ann Rheum Dis. 2016;75(9): 1583-94.

22. Novack SN, Pearson CM. Cyclophosphamide therapy in Wegener's granulomatosis. N Engl J Med. 1971;284(17):938-42.

23. Walsh M, Merkel PA, Peh CA, Szpirt WM, Puechal X, Fujimoto S, Hawley CM, Khalidi N, Flossmann O, Wald R, et al. Plasma exchange and glucocorticoids in severe ANCA-associated vasculitis. N Engl J Med. 2020;382(7):622-31.

24. Wilson JC, Sarsour K, Gale S, Petho-Schramm A, Jick SS, Meier CR. Incidence and risk of glucocorticoid-associated adverse effects in patients with rheumatoid arthritis. Arthritis Care Res (Hoboken). 2019;71(4):498-511.

25. Noel V, Lortholary O, Casassus P, Cohen P, Genereau T, Andre MH, Mouthon $L$, Guillevin L. Risk factors and prognostic influence of infection in a single cohort of 87 adults with systemic lupus erythematosus. Ann Rheum Dis. 2001:60(12):1141-4.

26. Ruiz-Irastorza G, Olivares N, Ruiz-Arruza I, Martinez-Berriotxoa A, Egurbide MV, Aguirre C. Predictors of major infections in systemic lupus erythematosus. Arthritis Res Ther. 2009;11(4):R109. 
27. Robson J, Doll H, Suppiah R, Flossmann O, Harper L, Hoglund P, Jayne D, Mahr A, Westman K, Luqmani R. Damage in the anca-associated vasculitides: long-term data from the European vasculitis study group (EUVAS) therapeutic trials. Ann Rheum Dis. 2015;74(1):177-84.

28. Watanabe H, Sada KE, Matsumoto Y, Harigai M, Amano K, Fujimoto S, Dobashi H, Yuzawa Y, Yamagata K, Muso E, et al. Rationale of concomitant cyclophosphamide for remission-induction in patients with antineutrophil cytoplasmic antibody-associated vasculitis: a propensity score-matched analysis of two nationwide prospective cohort studies. Mod Rheumatol. 2020:1-9. https://doi.org/10.1080/14397595.2019.1707997.

29. Lionaki S, Blyth ER, Hogan SL, Hu Y, Senior BA, Jennette CE, Nachman PH, Jennette JC, Falk RJ. Classification of antineutrophil cytoplasmic autoantibody vasculitides: the role of antineutrophil cytoplasmic autoantibody specificity for myeloperoxidase or proteinase 3 in disease recognition and prognosis. Arthritis Rheum. 2012;64(10):3452-62.

30. Lyons PA, Rayner TF, Trivedi S, Holle JU, Watts RA, Jayne DR, Baslund B, Brenchley P, Bruchfeld A, Chaudhry AN, et al. Genetically distinct subsets within ANCA-associated vasculitis. N Engl J Med. 2012;367(3):214-23.

\section{Publisher's Note}

Springer Nature remains neutral with regard to jurisdictional claims in published maps and institutional affiliations.

Ready to submit your research? Choose BMC and benefit from:

- fast, convenient online submission

- thorough peer review by experienced researchers in your field

- rapid publication on acceptance

- support for research data, including large and complex data types

- gold Open Access which fosters wider collaboration and increased citations

- maximum visibility for your research: over $100 \mathrm{M}$ website views per year

At BMC, research is always in progress.

Learn more biomedcentral.com/submissions 